\title{
Performance Study of Novel Median Filter for Image De-Noising
}

\author{
Geeta Hanji \\ PDA Engg.College \\ Gulbarga, India.
}

\author{
M.V.Latte \\ JSS Academy for Technical Education, \\ Bangalore, India.
}

\begin{abstract}
Most common method of clearing image noise is through median filtration. Median smoothers discard outliers (or impulses) quite effectively at lower noise densities but, fail to provide adequate smoothing for heavily noised images for the simple reason that, in the conventional median filter approaches, each and every pixel of an image is filtered without concern about healthy pixels. To suppress this deficiency, in this paper, an improved median filter by the name Novel Median Filter (NMF) is proposed to enhance the effectiveness of the median filter at higher noise situations to provide a detail preserving de-noising of digital images. Proposed NMF algorithm is a 3 -stage filter; first two stages are dedicated to selective switching median filtration in which noisy pixels are first detected and then the filtering operation is applied to restore their values with a valid (or a noise free) median computed for each $3 \times 3$ window position. Third level processing enables to remove any distortions present in the de-noised image. The proposed method, Novel Median Filter (NMF) is evaluated for its effectiveness in detail preserving de-noising capability on a large number of digital grey and color images affected with impulsive and non-impulsive noise in terms of objective and subjective quality measures. To better appraise the efficacy of the proposed method, Novel Median Filter (NMF), MATLAB simulation results in terms of peak signal to noise ratio (PSNR), mean square error (MSE) and the de-noised images are obtained and are compared with those obtained for a standard median filter (SMF) and its few variants and meaningful conclusions are derived. Detail preservation capability of the proposed filter has been demonstrated through experimentation on medical samples.
\end{abstract}

\section{General Terms}

Image Processing, Nonlinear Filter, Median Filtration.

\section{Keywords}

Novel Median Filter (NMF), Impulse Noise, Gaussian Noise, Valid Median.

\section{INTRODUCTION}

Images are rich information carriers and are often contaminated with noise of different types [1-2]. Fixed value (or salt and pepper) impulsive noise is one such noise that gets added with the digital images at random locations due to errors in transmission; pixel element's malfunctioning in the camera sensors, and faulty memory locations [13].Noise of this type possess an important and unique characteristic in that, it alters only a portion of the pixels' intensities at random positions into either relatively 'low' or 'high' intensity values enforcing those contaminated pixels with high intensity values to appear as white spots (or bright dots) on the image (that is 'salt'), while pixels noised with low grey values to appear as black spots (or dark dots) on the image (that is 'pepper'), while the rest are unaltered. Impulsive noise pixels of this nature, termed as salt-and-pepper noise (SPN) corrupted pixels [2-3] posses a relatively high contrast toward their neighborhood, even though the corruption percentage is low and can severely affect to degrade the appearance and the retrievation accuracy of the underlying image quite significantly [1-2] Further, it is to be noted that the human perception is heavily based on image edge information [1,3-4].

Therefore, the major essence and a prime goal in image restoration applications is to de-noise and reduce the salt and pepper impulsive noise effects [4-6] with vital features such as image edges preserved intact. This requirement can be met with some success using nonlinear basic median filter introduced by John Tukey [7] as an effective alternative to linear smoothers such as mean filters [1,2] for filtering signals having a wide spectrum. Median filtration is a good candidate for discarding the outliers and preserving the rapidly varying edge details [5-6]. However, the performance of the median filter is found to be inadequate in enhancing the images corrupted with high density impulsive noise and non-impulse noise [5]. Several variants of a median filter and its modifications have been reported in the literature for the removal of noise in digital images [8-17]. However, these median based filters are implemented uniformly across the image and as a result; both noisy and noise-free pixels get modified in the process of noise filtration. Consequently, effective suppression of impulse noise is accomplished at the cost of blurred and distorted features.

The use of switching scheme [21-25] in impulse noise suppression has attracted many researchers, because it can avoid the damage of healthy pixels by employing an impulse detector to discriminate between the noisy and healthy pixels and then replacing the affected pixel with a valid (or an appropriate) substitute. Such a decision based switching median filters [19, 21-25] are two-stage extensions and attempt to improve the efficacy of a standard median filter (SMF) [1] and its several variants. However de-noising results obtainable with these proposals are not much encouraging at higher noise situations. Work presented through this paper is an attempt to enhance the de-noising capability of SMF [1], AMF [15] and the method presented in [19] while incorporating a novel noise detection strategy to obtain an edge preserving de-noising performance at impulsive noise distributions as high as $80 \%$. 


\section{DESCRIPTION OF THE PROPOSED WORK}

Proposed Novel Median Filter is a selective switching median filter and is based on SF paradigm shown in Figure (1) and aims primarily at improving the filtering efficacy of the Standard Median Filter (SMF) [1] at high noise situations and an Adaptive Mask Median Filter (AMF) [15] at very high ( $>70 \%$ ) noise density situations.

In a standard median filter there is no means to check whether the computed median is an impulse or not. In adaptive mask

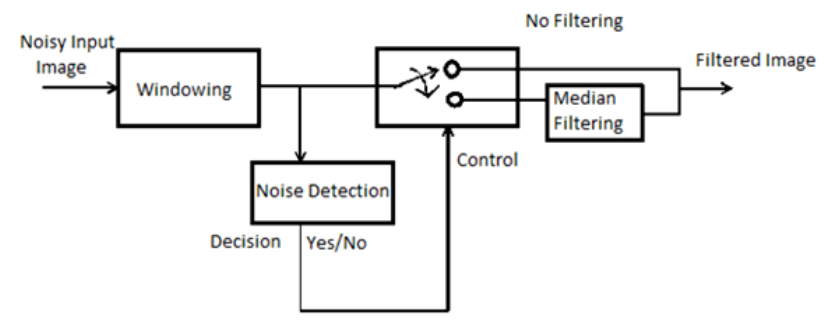

Fig 1: Switching Median Filter (SMF) paradigm

median filter (AMF), though the mask size is increased until it reaches the maximum value to provide the noise free median, it doesn't guarantee to provide good results at very high noise density situations for the simple reason that, all most all the pixels are contaminated and the noise still persists. Moreover, AMF is too time consuming and complex. In contrast to SMF, variants of a median filter $[8,11$ 12,14,17], and Adaptive Median Filter (AMF) [15], the proposed novel median filter (NMF) is quite simple and attempt to produce noise free and distortion less de-noised images with the image details preserved intact. Novel Median Filter (NMF) is a three- stage filtration method; first two levels are dedicated to noise detection and estimation of a valid median term for noised pixel replacement, where as the third level of processing aims at suppressing any distortions present in the de-noised output. Proposed method NMF is basically a decision based selective switching median filter technique in which each pixel undergoes an impulse noise check process and is based on the computation of a valid (or a noise free) median for each $3 \times 3$ window position while looking for a better representative for noised pixel replacement if an appropriate value of the mask median is not found. This is an ideal property of substructure and is exercised to obtain a valid median to substitute for a noised pixel.

\subsection{Proposed Novel Median Filter (NMF) Algorithm \\ NMF algorithm is explained in the following steps.}

Step-1) Select the working window of size $3 \times 3$, and compute the window median $\mathrm{M}_{\mathrm{W}}$.

Step-2) Test the median $\mathrm{M}_{\mathrm{W}}$ to verify if it is an impulse or not by checking its value against minimum (0) and maximum (255).That is,

$$
\begin{array}{r}
\text { IF } 0<\mathrm{M}_{\mathrm{W}}<255 \\
\text { GOTO step } 3
\end{array}
$$

ELSE GOTO step 4.
Step-3) Declare $M_{W}$ as a valid median value and replace the reference, center noisy pixel by the grey value of $\mathrm{M}_{\mathrm{W}}$.

Step-4) If the computed window median $\mathrm{M}_{\mathrm{W}}$ is an impulse (that is, $\mathrm{M}_{\mathrm{W}}=0$ or 255 ): In this case a pixel with its value between the minimum and a maximum values is searched for, in the neighborhood of the median position. When it is realized that more than half (or 50\%) of the pixels in the window are impulses, then the new noise exclusive median $\mathrm{M}_{\mathrm{NE}}$ is computed as follows:

$$
\mathrm{M}_{\mathrm{NE}}=\frac{\mathrm{X}_{\min }+\mathrm{M}_{\mathrm{W}}+\mathrm{X}_{\max }}{3}
$$

where $\mathrm{X}_{\min }, \mathrm{M}_{\mathrm{W}}$ and $\mathrm{X}_{\max }$ are the lowest, the middle and the highest pixel intensity values in the sorted array from the current window. This newly computed median value $\mathrm{M}_{\mathrm{NE}}$ is referred to as a valid (or a noise free) median.

Step-4) Inspect and replace the noisy pixels with a newly computed median value $\mathrm{M}_{\mathrm{NE}}$. This is the stage of impulse noise rejection at medium and high noise situations.

Step-5) Re-sort all the pixels in the window, and the processing is now towards removing any distortions present. Processing at this stage is always done, even when no impulse noise is detected in the current window position. Processing starts with the adjacent pixel differences $\mathrm{D}_{\mathrm{AP}}$ being computed as:

$$
\mathrm{D}_{\mathrm{AP}}=\left(\mathrm{X}_{\mathrm{i}+2}\right)-\left(\mathrm{X}_{\mathrm{i}+1}\right)
$$

for all $i=1,2 \ldots .2 n-2$, where $N^{2}=2 n+1$.

and

$$
\begin{aligned}
& \left(D_{A P}\right)_{\max }=\operatorname{Max}\left(D_{A P}\right) \\
& \text { for all ' } i \text { ', where } 1 \leq i \leq 2 n-2 .
\end{aligned}
$$

Once the $\left(\mathrm{D}_{\mathrm{AP}}\right)_{\max }$ is computed, it is evaluated by the following computations for values at the extreme ends of the sorted list :

$$
\begin{aligned}
& P_{1}=\left\{\begin{array}{cc}
M_{N E}, & \text { if }\left(X_{2}-X_{1}\right)>D_{A P(\max )} \\
X_{1} ; & \text { otherwise }
\end{array}\right. \\
& P_{2 n+1}=\left\{\begin{array}{cc}
M_{N E}, \text { if }\left(X_{2 n+1}-X_{2 n}\right)>D_{A P(\max )} \\
X_{2 n+1} ; & \text { otherwise }
\end{array}\right.
\end{aligned}
$$

\subsection{Illustration of NMF Algorithm.}

\begin{tabular}{|c|c|c|}
\hline 20 & 30 & 40 \\
\hline 60 & 0 & 255 \\
\hline 50 & 70 & 220 \\
\hline
\end{tabular}

Fig 2: Part of image pixels contained within a $3 \times 3$ input window ( $3 \times 3$ sub image pixels)

Assuming the sub image shown in Figure (2), the proposed NMF algorithm is illustrated below.

\subsubsection{Stage 1}

i) All pixels in the window are arranged in the increasing order to obtain the sorted array as below.

$$
0,20,30,40,50,60,70,220,255 \text {. }
$$

ii) From the above sorted array, compute the median value of the window $\mathrm{M}_{\mathrm{W}}$. That is $\mathrm{M}_{\mathrm{W}}=50$.

iii) Computed median satisfies the condition; $0<\mathrm{M}_{\mathrm{W}}<255$. 
Thus we confirm ' $\mathrm{M}_{\mathrm{W}}=50$ ' as a valid and noise free (or an appropriate) median in stage 1 processing.

\subsubsection{Stage2}

Numbers of impulse affected pixels in the sub image are counted in this stage. In this illustrative example two (that is, less than $50 \%$ of the total number of pixels in the sub image) impulses (' 0 ' and ' 1 ') are detected and are replaced with the median ' $\mathrm{M}_{\mathrm{W}}$ ' computed in the previous stage to obtain the following array of pixels.

$$
50,20,30,40,50,60,70,220,50 .
$$

\subsubsection{Stage3}

i)All the pixels in the sub image are re-sorted again in an increasing order prior to processing in the third and a final stage and the processing is done as explained in the next step.

ii)By considering the re-sorted array $(20,30,40,50,50,50,60,70,220)$, starting from the pixel in the second place and ending at the place 'last but one' (that is, eighth place pixel), adjacent pixel differences ' $\mathrm{D}_{\mathrm{AP}}$ ' are obtained as follows : $(40-30)=10,(50-40)=10,(50-50)=0$, $(50-50)=0$, and $(60-50)=10,(70-60)=10$. Maximum difference values ' $\left(\mathrm{D}_{\mathrm{AP}}\right)_{\max }$ ' is found to be equal to ' 10 '.

iii) In order to restore back any other distorted pixel values, deviations between adjacent pixel values in the re-sorted array are compared with the maximum difference value $\left(\mathrm{D}_{\mathrm{AP}}\right)_{\max }$ explained as follows.

Considering the difference between the first and the second pixel values in the re-sorted list i.e.30-20='10' is not greater than $\left(\mathrm{D}_{\mathrm{AP}}\right)_{\max }$. Pixel value '20' is thus not altered. However, the difference between last two pixels $220-70=150$ is larger than $\left(\mathrm{D}_{\mathrm{AP}}\right)_{\max }$ and thus the pixel value ' 220 ' is substituted with median value $\mathrm{M}_{\mathrm{W}}={ }^{\prime} 50$ '. Sub images in Figure (3) indicate the restoration operations.

\begin{tabular}{|c|c|c|c|c|c|c|c|c|}
\hline \multicolumn{3}{|c|}{$\begin{array}{c}\text { Noisy sub } \\
\text { image }\end{array}$} & \multicolumn{2}{c|}{$\begin{array}{c}\text { Median filtered image } \\
\text { (stage 1 and 2) }\end{array}$} & \multicolumn{3}{c|}{$\begin{array}{c}\text { Finally processed } \\
\text { image (stage 3) }\end{array}$} \\
\hline 20 & 30 & 40 & 20 & 30 & 40 & 20 & 30 & 40 \\
\hline 60 & $\mathbf{0}$ & $\mathbf{2 5 5}$ & 60 & 50 & 50 & 60 & 50 & 50 \\
\hline 50 & 70 & 220 & 50 & 70 & $\mathbf{2 2 0}$ & 50 & 70 & 50 \\
\hline
\end{tabular}

Fig 3: Illustration of proposed filter NMF.

\section{EXPERIMENTAL RESULTS AND DISCUSSION}

Performance of the proposed method, Novel Median Filter (NMF) and other filters are accessed through MATLAB (R2008a, version7.6) simulation results in terms of terms of Peak Signal to Noise Ratio (PSNR) and Mean Square Error (MSE) results (equation (6) and (7)). For a large number of images from the author's data base, simulation results obtained with SMF [7], WMF [11-12],CWMF [14], AMF
[15], PSMF[17], and IMF[19] in terms of Peak Signal to Noise Ratio (PSNR) for Lena $(512 \times 512$ grey) image are graphically shown in Figure (4).

$$
\begin{gathered}
\mathrm{PSNR}=10 * \log _{10} \frac{255^{2}}{\mathrm{MSE}} \\
\mathrm{MSE}=\frac{1}{\mathrm{MN}} \sum_{\mathrm{i}=1}^{\mathrm{M}} \sum_{\mathrm{j}=1}^{\mathrm{N}}[\mathrm{O}(\mathrm{i}, \mathrm{j})-\mathrm{Y}(\mathrm{i}, \mathrm{j})]^{2}
\end{gathered}
$$

where ' 255 ' is the maximum grey level in the image, $\mathrm{O}(\mathrm{i}, \mathrm{j})$ and $Y(i, j)$ are the original noise free image and the denoised images (each of dimension $\mathrm{M} \times \mathrm{N}$ ) respectively. The MSE and PSNR values obtained for the filtered image indicates the high performance of the proposed filter method at noise densities around $90 \%$ compared to those obtained with Adaptive Median Filter (AMF) and Improved Median Filter (IMF).At noise density situations upto $30 \%$, the performance of the proposed filter is not much encouraging compared to all other filters considered in our work for comparison. However an improvement in denoising performance in terms of PSNR and MSE has been observed compared to SMF, PSMF, Med-2, WMF and CWMF at increased noise density situations (> 35\% up to $90 \%$ ).

De-noising performance of the proposed filter NMF, at $80 \%$ salt and pepper noise for Lena (512x 512 grey) image shown in Figure (6) in comparison with the SMF[1], WMF[12], Median-2 (Median filter with threshold) [1], AMF[15], IMF[17] exhibit its efficacy in addressing highly noised images with visually pleasing results. Special application of the proposed filter in addressing the simultaneous suppression of the mixed noise namely, mixed Gaussian-Impulse noise (MGIN) with good edge preservation capability has been demonstrated through the results presented in Figures (7) and (8). The performance of the proposed filter in suppressing impulse noise from the color images illustrated through de-noising Lena $(512 \times 512$ color image) in Figure (9a-9y) are quite acceptable and pleasing.

From the qualitative results obtained for a large number, and a variety of images such as low detail images (ex. Cameraman for which results are not shown in this paper due to space limitations), medium detail images such as Lena, and high detail images such as Brain Tumor image, it can be honestly concluded that, the proposed NMF is quite effective in de-noising low, medium as well as high detail images affected with salt and pepper noise up to $80 \%$ noise densities with image edge details preserved intact.

\section{CONCLUSIONS}

An efficient 3- stage, selective switching median filter by the name Novel Median Filter (NMF) for image de-noising application is presented and its special application in denoising Brain tumor images contaminated with a mixed Gaussian -impulse noise (MGIN) is also illustrated. Proposed NMF shows consistent and stable performance across a wide range of noise densities varying from $10 \%$ to $80 \%$. 


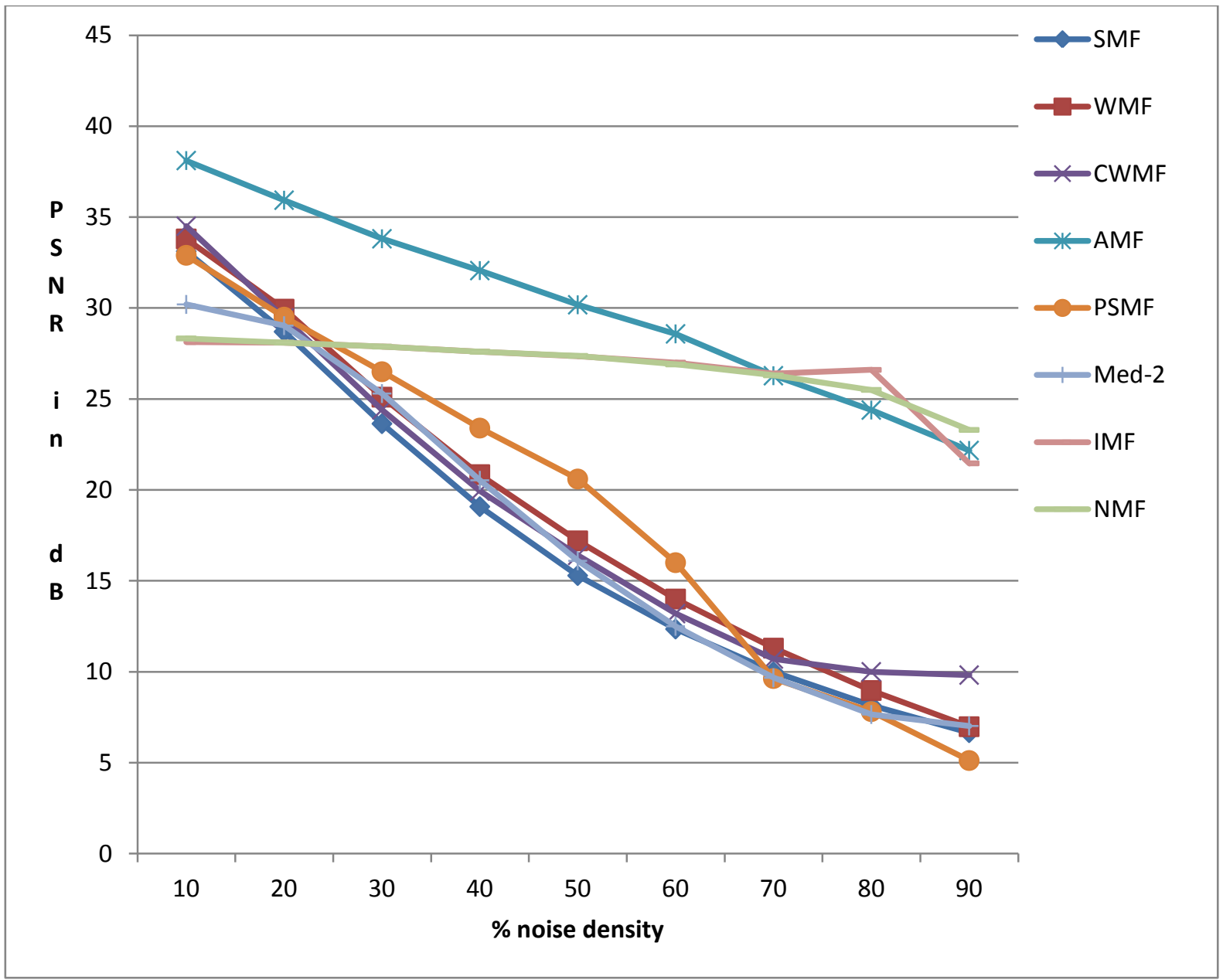

Fig 4: Performance comparison of the proposed method NMF and other filters in terms of PSNR

Performances of the proposed filter are verified with the images contaminated with SPN alone and with both SPN and AWGN on grey and color images and satisfactory edge preserving de-noising performance has been realized. For the future, it will be interesting if the proposed filter algorithm, Novel Median Filter (NMF) is suitably modified and performed over both grey and color images noised with random valued impulsive noise densities.

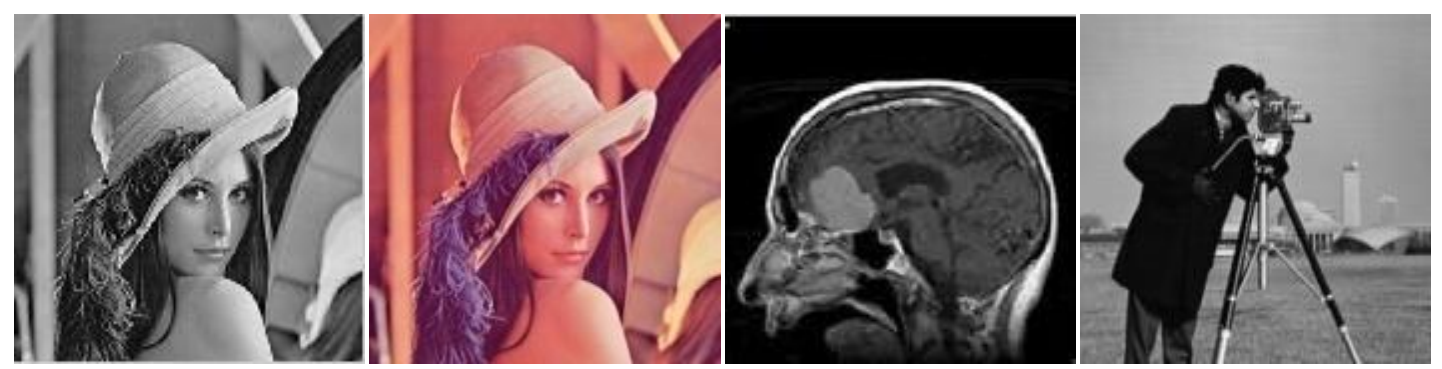

Fig 5: Few original test images from the author's data base. 


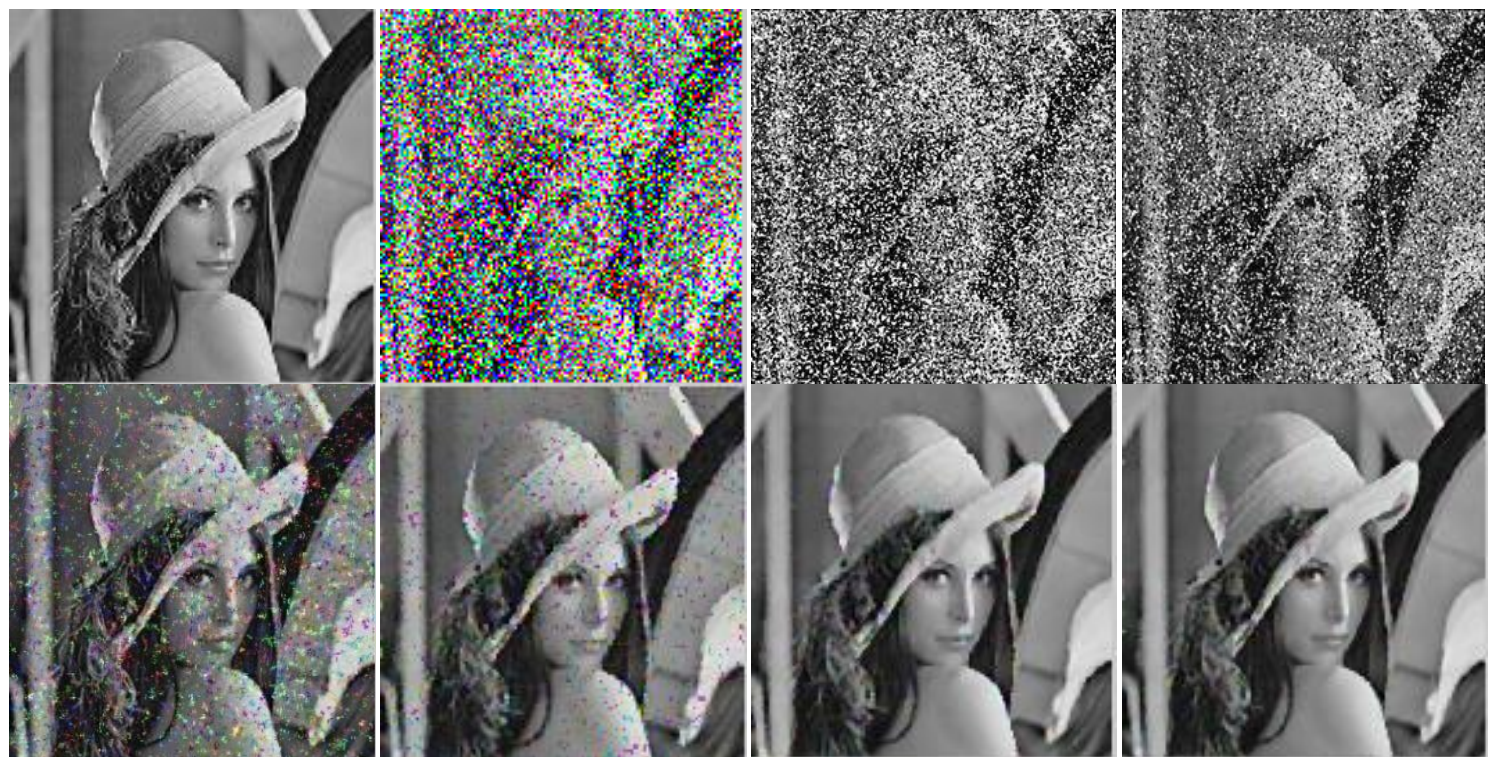

Fig 6: De-noising performance at $80 \%$ salt and pepper noise for Lena $(512 \times 512$ grey) image. (a)Original (b) Noised. De-noised with (c) SMF [1] (d) WMF [11-12] (e) Med-2[1] (f) AMF [15] (g) IMF [19] (h) Proposed NMF.
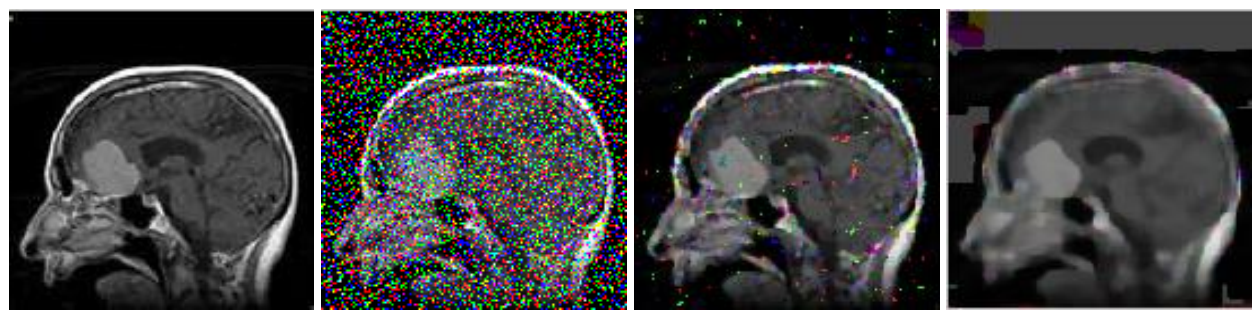

Fig 7: (a) Original Brain Tumor image, (b) Noised with $30 \%$ SPN+AWGN at 0.1 Variance. Restored with (c) SMF (d) NMF.
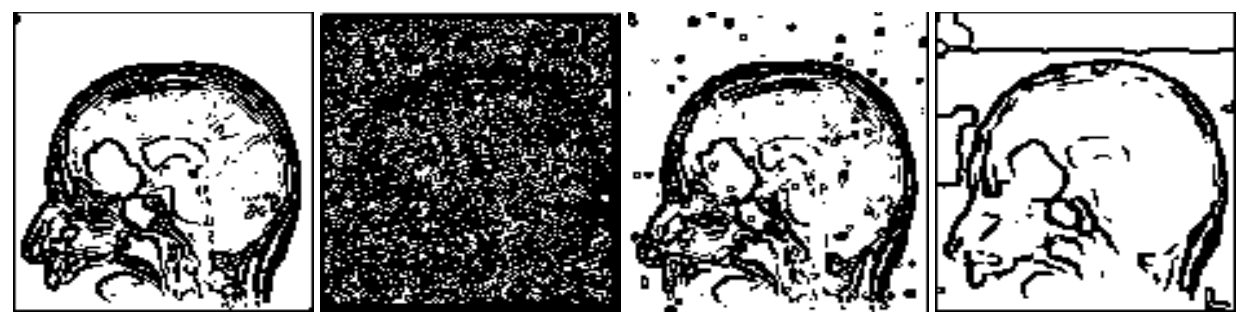

Fig 8: Corresponding edge detected images.

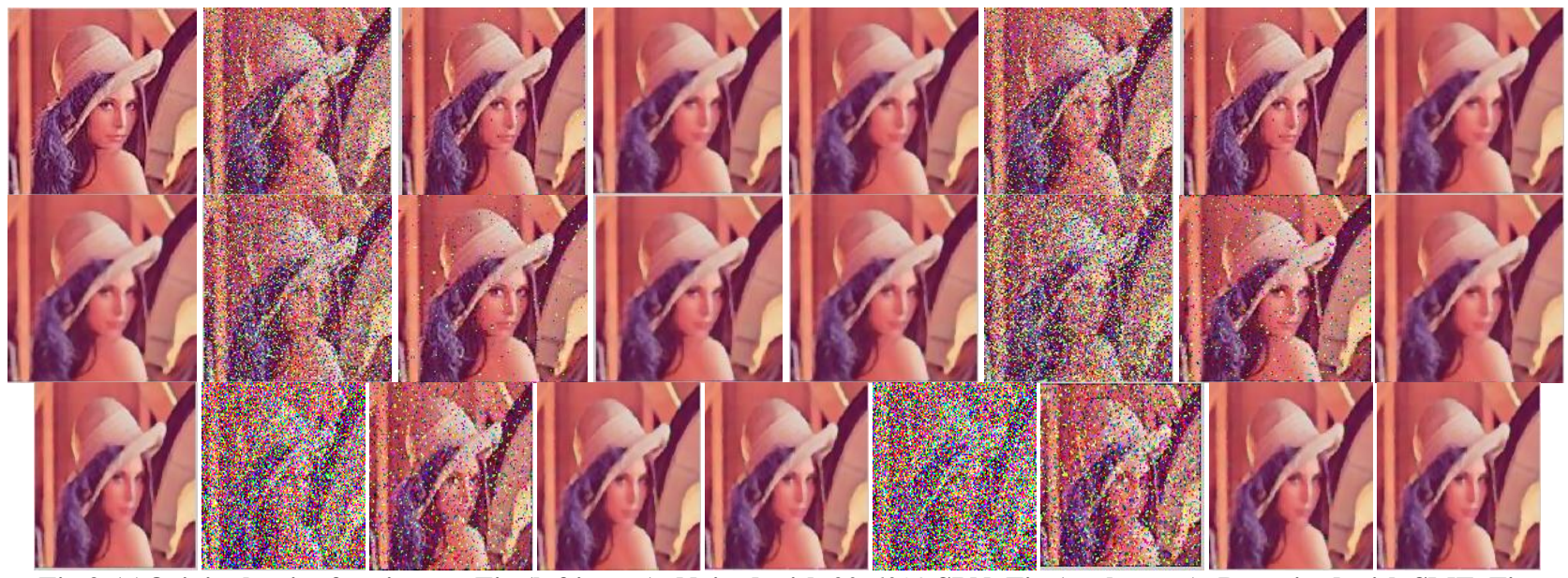

Fig 9:(a)Original noise free image. Fig.(b,f,j,n,r,v): Noised with 20-60\% SPN. Fig (c,g,k,o,s,w): De-noised with SMF. Fig $(d, h, l, p, t, x)$ : De-noised with IMF[19]. Fig (e,i,m,q,u,y): De-noised with NMF. 


\section{REFERENCES}

[1] Gonzalez R.C. and Wood R.E.: Digital Image Processing, PHI, Second Edition, (2007).

[2] Pitas I. and Venetsanopoulos A. N.: Nonlinear Digital Filters: Principles and Applications, Boston, MA: Kluwer Academic, (1999)

[3] Bovik A.C: Hand book of Image and Video Processing, Academic Press, (2000).

[4] Ataman E., Aatre V.K. and Wong K.M., Some Statistical Properties of Median Filters, IEEE Trans. Acoust., Speech and Signal Processing, Vol. 29, No. 5, (1981), pp. 1073-1075.

[5] Nodes T.A. and Gallagher N.C., Median Filters: Some Modifications and Their Properties, IEEE Trans. Acoust. Speech and Signal Processing, Vol. 30, No. 5, (1984), pp. 739-746.

[6] Bovik A.C., Huang T.S., and Munson. D.C., The Effect of Median Filtering on Edge Estimation and Detection, IEEE Trans. Pattern Anal. Machine Intelligence, Vol. PAMI-9, No.2, (1987), pp. 181-194.

[7] Tukey J.W.: Exploratory Data Analysis Reading, Addison Wesley.in, (1971).

[8] Sin Hoong Teoh, Boon Tatt Koik, and Haidi Ibrahim, Exploration of Current Trend on Median Filtering Methods Utilized in Digital Grayscale Image Processing, International Journal of Materials, Mechanics and Manufacturing, Vol. 1, No.1, (2013).

[9] Nieminen A., Heinonen P., and Neuvo Y., A New Class of Detail Preserving Filters for Image Processing, IEEE Trans. Pattern Anal. Machine Intell., Vol. PAMI-9, No.1, (1987), pp. 74-90.

[10] Arce G.R. and Foster R.E., 'Detail Preserving RankedOrder based Filters for Image Processing', IEEE Trans. Acoustic., Speech, Signal Process., Vol. 37, No.1, (1989), pp. 83-98.

[11] Brownrigg D.R.K., The Weighted Median Filter, Commun. ACM, Vol. 27, No.8, (1984), pp. 807-818,

[12] Yin L., Yang R., Gabbouj M. and Neuvo Y., Weighted median filters: A Tutorial, IEEE Trans. Circuits and Systems-II: Analog and Digital Signal Processing, Vol.43, No.3, (1996), pp.157-192.

[13] Astola J. and Kuosmanen P.: Fundamentals of Nonlinear Digital Filtering: Boca Raton, FL: CRC (1997),

[14] Ko, S.J., and Lee, Y. H., Center Weighted Median Filters and Their Applications to Image Enhancement,
[15] IEEE Trans. Circuits and Systems, vol. 38, (1991), No. 9, pp. 984-993.

[16] H.Hwang and R. A.Haddad, Adaptive Median Filters: New Algorithms and Results, IEEE Trans. Image Processing, Vol. 4, (1995), pp. 499-502.

[17] Tao Chen and Hong Ren, Space Variant Median Filters for the Restoration of Impulse Noise Corrupted Images, IEEE Trans. Circuits and Syst-II, Analog and Digital Signal Processing, Vol.48, No.8, (2001), pp.784-789.

[18] Wang Z. and Zhang D., Progressive Switching Median Filter for the Removal of Impulse Noise from Highly Corrupted Images, IEEE Trans. on Circuits and Systems II, Vol. 46, No.3, (1999), pp. 78 - 80.

[19] T. Chen and H. R. Wu, Adaptive Impulse Using CenterWeighted Median Filters, IEEE Signal Processing Letters, (2001), pp.1-3.

[20] Kwame Boateng, Ben Weyori Asubam and David Sanka Laar, Improving the Effectiveness of the Median Filter, International Journal of Electronics and Communication Engineering, Vol. 5, No. 1, (2012), pp. 85-97.

[21] Pragati Agarwal, Jayendra Singh Verma, A Survey of Linear and Non-Linear Filters for Noise Reduction, International Journal of Advanced Research in Computer Science and Management Studies ,Vol.1,(2012).

[22] Sonali R. Mahakale, Nilesh Singh V.T., A Comparative Study of Image Filtering on Various Noisy pixels, International Journal of Image Processing and Vision Sciences, Vol.1, (2012).

[23] Priyanka Kamboj, Versha Rani, Brief Study of Various Noise Model and Filtering Techniques, Journal of Global Research in Computer Science, Volume 4, No. 4, (2013).

[24] S. Zhang and M. A. Karim, A New Impulse Detector for Switching Median Filters, IEEE Signal Process. Letters, Vol. 9, No. 11, (2002), pp. 360-363.

[25] Geeta Hanji, M.V.Latte, A New Impulse Noise Detection and Filtering Algorithm, Image Processing \& Communications (IPC), Vol. 16, No. 1-2, (2012), pp.4348 .

[26] Chung-Chia Kang, Wen-June Wang, Modified Switching Median Filter with One More for Detector for Impulse Noise Removal, Int. J. Electron Commun. (AEU), Vol.63, (2009), pp.998-1004. 